\title{
Accuracy of Pharmacy Benefit Manager Medication Formularies in an Electronic Health Record System and the Epocrates Mobile Application
}

\author{
Miranda R. Andrus, PharmD, BCPS, FCCP; Jeanne B. Forrester, PharmD; Kenda E. Germain, PharmD; \\ and Lea S. Eiland, PharmD, BCPS, FASHP
}

\begin{abstract}
BACKGROUND: Physicians commonly use formulary medication coverage information generated by electronic heath records (EHRs) and the Epocrates mobile drug database application when making medication selection decisions. Nonformulary selections may lead to higher outof-pocket patient costs and nonadherence with prescribed regimens. Nonformulary selections also contribute to higher overall health plan spending. However, the accuracy of these systems compared with actual insurance coverage is not known.
\end{abstract}

OBJECTIVE: To assess the accuracy of formulary status icons generated by an EHR system and the Epocrates mobile application for patients with Alabama Medicaid and Blue Cross Blue Shield of Alabama (BCBS), the primary insurance providers in the state of Alabama.

METHODS: Patients of all ages who had a chart review performed at the outpatient family medicine or pediatric clinics at the University of Alabama at Birmingham Huntsville Medical Regional Campus from May to October 2013 were included in this retrospective analysis. Patients who were not insured by either Alabama Medicaid or BCBS were excluded. Patients who did not have new medications added at the time of the visit were also excluded. For each medication prescribed, the formulary status provided in the EHR system and Epocrates was compared with the actual Medicaid and BCBS formularies published online, and the accuracy of the 2 databases was determined.

RESULTS: A total of 1,529 medication records were analyzed. The EHR and Epocrates provided accurate formulary information for $93.1 \%$ and $89.4 \%$ of medications, respectively. Formulary information generated by the EHR was $96.3 \%$ accurate for Medicaid patients and $80.1 \%$ accurate for BCBS patients. Epocrates was $88.2 \%$ accurate for Medicaid patients and $94.4 \%$ accurate for BCBS patients. A total of 936 medication records from the pediatric clinic were analyzed, and the majority of these patients $(88.4 \%)$ had Medicaid insurance. In this population, the EHR was more accurate $(96.9 \%)$ than Epocrates (86.6\%). Of the small number of pediatric medication records $(n=109)$ associated with patients who had BCBS, Epocrates was more accurate $(92.7 \%)$ than the EHR (83.5\%). In family medicine, 593 medication records were analyzed. Again, for Medicaid patients, the EHR was more accurate $(95.3 \%)$ than Epocrates (91.5\%). For those with BCBS, Epocrates was more accurate (95.3\%) than the EHR (78.2\%). When overthe-counter (OTC) medications $(n=232)$ were analyzed separately from prescription medications, it was found that overall the EHR was $90.5 \%$ accurate, and Epocrates was $41.4 \%$ accurate. It is important to note that when only prescription medications were analyzed, the accuracy rates were high (93.6\% for the EHR and $98.0 \%$ for Epocrates).

CONCLUSIONS: Formulary information generated by the EHR system and the Epocrates mobile application is a useful tool for physicians when prescribing medications, but neither source is completely accurate. Prescribers should be particularly cautious when making OTC formulary decisions using the Epocrates mobile application, since the formulary information provided for these medications was found to be the least accurate.

\section{J Manag Care Spec Pharm. 2015;21(4):281-86}

Copyright $\odot 2015$, Academy of Managed Care Pharmacy. All rights reserved.

\section{What is already known about this subject}

Accessing formulary medication information through e-prescribing systems can improve efficiency when the formulary information is up to date and accurate.

Systems with formulary decision support can influence prescribers to prescribe medications that may be less expensive for patients.

Selecting formulary medications decreases patients' financial burdens and may lead to better patient adherence.

\section{What this study adds}

Formulary information in the EHR system and the Epocrates application is relatively reliable in an outpatient population, with 93.1\% and $89.4 \%$ accuracy, respectively.

Formulary information for over-the-counter medications was less reliable, with $90.5 \%$ accuracy for the EHR, and only $41.4 \%$ accuracy for Epocrates.

D rescribers commonly use formulary medication coverage information generated by electronic health records (EHRs) with formulary decision support and mobile drug database applications when making medication selection decisions. Nonformulary selections may lead to higher patient costs and nonadherence to prescribed regimens, as well as higher overall health plan spending. Selection of nonformulary medications can also increase the workload of medical staff members due to more contact needed with dispensing pharmacies and paperwork generated by prior authorization approvals. Evidence from previous clinical trials support that selection of formulary medications leads to better patient adherence, possibly due to lower cost to the patient. ${ }^{1}$ Nonformulary selections also may lead to higher overall health plan spending. Electronic 
prescribing (e-prescribing) systems with integrated decision support, including access to formulary information, and utilization management (i.e., prior authorizations and step therapy) can influence prescriber behavior and may result in lower prescription medication cost. ${ }^{2}$ These findings, along with the continued emphasis on the need to decrease costs in all areas of health care, highlight the importance of verifying the accuracy of electronic formulary sources.

Data support the positive outcomes realized by physicians with direct access to formulary information. Lapane et al. (2011) reported that clinicians and their staff found that e-prescribing systems with formulary medication information improved efficiency but also cited inefficiencies of the system when formularies were not kept up to date or were incorrect. ${ }^{3}$ Participants in this study were evaluated using a combination of surveys, interviews, and focus groups and consistently identified that having access to formulary information saved the prescriber time by decreasing the correspondence with pharmacies related to prescription medication cost or lack of coverage, as well as allowing them to be aware of prior authorization requirements at the time of prescribing the medication. In addition, the providers stated that having the formulary information allowed them to better consider the financial needs of the patient. In another trial, Fischer et al. (2008) reported that clinicians with access to systems providing formulary decision support were more likely to prescribe less expensive medications and that this practice could lead to significant cost savings when considering the number of prescriptions processed annually. ${ }^{4}$ In contrast, a study by Ross et al. (2005) compared the outcomes of formulary access in an e-prescribing system with traditional prescribing practices without electronic access for providers within a managed care organization. ${ }^{5}$ Formulary status was indicated for preferred first tier, second tier, and nonformulary medications. There was no difference found in the level of formulary prescribing between the electronic prescribers and traditional prescriptions. It is interesting to note that in this study $25 \%$ of traditional prescribers reported using Epocrates as a source for formulary information.

Incomplete and inaccurate formulary information has been a cause of skepticism and underutilization of formulary decision support by providers. Crosson et al. (2012) published data that described challenges with formulary checks in e-prescribing systems, which led to physician distrust and unwillingness to rely on formulary information. ${ }^{6}$ While some prescribers in the study found the information helpful and claimed to use it regularly, others were frustrated by incomplete or potentially inaccurate data. The latter were unlikely to use the formulary and benefit information provided and continued to rely on pharmacies to notify them of insurance coverage problems. In a similar study that surveyed prescribers about formulary information in e-prescribing systems, there was no significant difference perceived in the volume of calls about drug cover- age problems with e-prescribing, and many thought that the formulary information was often incomplete. ${ }^{7}$

In 2008, the Centers for Medicare \& Medicaid Services issued standards for e-prescribing under Medicare Part D. These rules require the use of formulary and benefit information for e-prescribing. However, the accuracy of such systems has not been addressed. Accuracy is vitally important in the context of multiple formularies that are continually changing, the release of new medications, and changes to generic status.

The institution participating in this study, The University of Alabama at Birmingham Huntsville Regional Medical Campus, uses the Allscripts TouchWorks EHR system (http://www. allscripts.com/en/solutions/ambulatory-solutions/ehr/Show/ ProductSelect/AllscriptsEnterpriseEHR/Overview.html), and by our observations, physicians in our practice tend to consult the Epocrates mobile application (http://www.epocrates.com) for formulary guidance. The accuracy of the formulary information found in the EHR and Epocrates mobile application is unknown. The accuracy of these selection tools has not been systematically verified in our patient population.

The purpose of this study was to assess the accuracy of insurance formulary status icons generated by the EHR system and coverage information found in the Epocrates mobile application for patients with Alabama Medicaid and Blue Cross Blue Shield (BCBS) of Alabama, the primary insurance providers in the state of Alabama, and to compare the accuracy of both sources to determine the most accurate electronic reference to use in our patient population.

\section{Methods}

\section{Study Site}

The Huntsville Regional Medical Campus includes a family medicine outpatient clinic and a pediatric clinic, which were used for this study. The family medicine outpatient clinic is staffed by approximately 36 family medicine residents and 8 attending physicians with an average of 2,300 patient encounters per month. The patient population is varied and includes obstetric, pediatric, and adult patients. The insurance mix consists of 34\% Medicare, 34\% Medicaid, 28\% private insurance, and $4 \%$ uninsured. The pediatric outpatient clinic is staffed by 4 attending physicians with an average of 1,100 patient encounters per month. The patient population includes all children up to age 18. The insurance mix consists of $68 \%$ Medicaid, 29\% private insurance, and 3\% uninsured. The family medicine and pediatric clinics have been using the EHR system since 2006, which displays the patient's primary insurance coverage in the patient information bar at the top of each screen. Fourth-year student pharmacists are assigned to these clinics for 5-week advanced pharmacy practice clinical rotations. Students actively see patients in the clinics approximately 4 to 6 half-days per week during rotations. Data were collected retrospectively from the EHR for patients of all ages seen at the family medicine and 
pediatric clinics by pharmacy students on clinical rotations. Data collection occurred from May to November 2013.

The EHR system used at our institution generates icons indicating the status of a medication on the formulary of the patient's insurance. The icons appear in the system as smiley faces of green, yellow, or red color. A green face indicates that the medication is covered by the patient's insurance; yellow indicates that a prior authorization is required; and red indicates that the medication is not covered by the patient's prescription insurance plan. Formulary information is provided to the EHR directly from insurance providers; however, it is unknown how frequently updates are made. When patients arrive for visits, the computer system updates formulary information for their medications. The icons are available on patients' profiles throughout the day of their scheduled appointments, and it is presumed this information is updated with current coverage status upon every patient visit. The physicians generally assume that the formulary information provided via the EHR is accurate and commonly use this information when making prescribing decisions.

The physicians also commonly use the Epocrates mobile application for prescribing and formulary information. The Epocrates mobile application is available as a free download for smartphones and also provides formulary coverage information for medications. Formulary coverage information is generated by setting the desired insurance provider and selecting the medication profile for the drug being prescribed. The coverage information is displayed on the medication profile under the formulary tab and appears in written format describing the coverage status. For Alabama Medicaid, formulary status is described as "covered drug" or "prior authorization required." For Alabama BCBS, formulary status is described as "tier 1 , generic"; "tier 2, intermediate copay"; and "tier 3, high copay." The individual insurance providers manage updates to formulary information directly through a web-based portal rather than Epocrates being responsible for updating this coverage information. ${ }^{9}$ The insurance providers can access the portal at any time, so the frequency of formulary updates varies with each insurance plan.

\section{Population}

The population was selected based on third-party coverage of Medicaid or BCBS. Additionally, patients were eligible only if they were seen at the family medicine and pediatric clinics by pharmacy students on clinical rotations. Patients who were uninsured or insured by third-party payers other than Medicaid and BCBS at the time of their clinic visits were not included in the study. Patients who were seen in the clinics but did not have home medications or new medications prescribed at their visits were also excluded. Medicaid and BCBS were selected, since these are the major insurance providers in Alabama and combined cover the majority of the patients seen in the clinics. Medicare was excluded due to the complexity of multiple
Medicare Part D prescription plans, since the specific Medicare Part D plan is not noted in the patient's chart, so formulary status could not be obtained from the EHR.

The Alabama Medicaid Agency is jointly funded by federal and state governments and provides medical assistance for certain individuals and families with low income and resources. In 2010, enrollment exceeded 1 million beneficiaries, and about $11 \%$ of total expenditures is currently spent on pharmacy services. ${ }^{10,11}$ Copays for prescription drugs range from $\$ 0.65$ to $\$ 3.90$ per prescription; however, those who are under aged 18 years, pregnant, or receiving family planning services do not have to pay a copayment. ${ }^{12}$ The formulary is specific for which prescription medications are covered, and restrictive with only a few brand-name products covered. It is not a tiered system. The formulary is updated every 3 months. Generic over-thecounter (OTC) medications were generally covered for adults and pediatric patients during the time of this study.

BCBS of Alabama has provided individual and group health plans since 1936 and currently has overall 3 million enrollees in over 28,000 companies..$^{13}$ The prescription benefit is tiered, with 3 copayment levels: generic drugs (tier 1), preferred brand drugs (tier 2), and nonpreferred brand drugs (tier 3). Copayment levels vary depending on the plan. ${ }^{14}$ Most prescription medications are covered in these tiers, with a few exclusions and restrictions for specialty products. Generally, OTC products are not covered, and the formulary is updated "regularly" per the company.

\section{Procedure}

For each chart reviewed, the medication and dosage form prescribed, date seen in clinic, third-party payer (Medicaid or BCBS), and medication coverage status was collected from the EHR. Coverage status was defined as covered, prior authorization required, or not covered. The status provided in the EHR was then compared with the actual third-party formularies published online on the Alabama Medicaid and BCBS of Alabama websites at the time of the patient visit. The coverage information in the Epocrates mobile application for all the medications gathered from the EHR was also collected and compared with the published online formularies. Determination of whether the EHR or Epocrates was more accurate for the population was based on their actual published third-party coverage information. Comparison of the 2 sources to determine the most accurate source for each population was completed. The primary outcome was the percentage accuracy of the EHR and the Epocrates mobile application when compared with the actual online formulary.

All data were collected and entered into Microsoft Excel (Microsoft Corporation, Redmond, WA). Descriptive statistics were used to analyze the data. All results are expressed as proportions. 


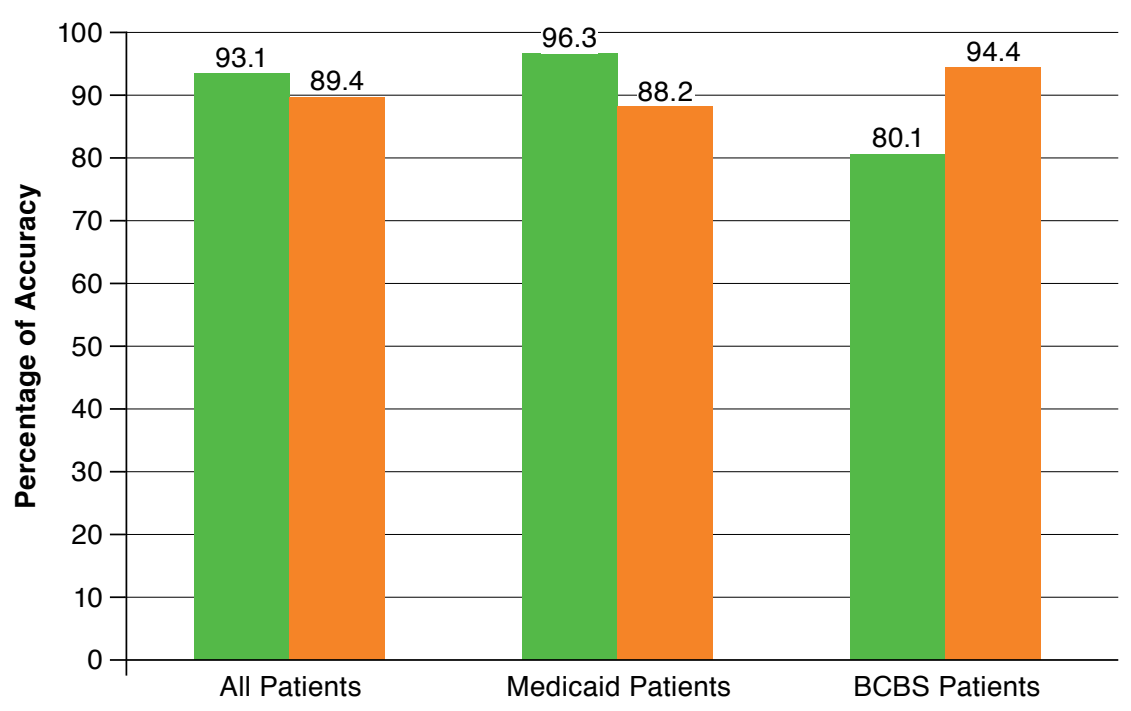

EHR
Epocrates

BCBS = Blue Cross Blue Shield; EHR=electronic health record.

\section{Results}

\section{All Patients}

A total of 1,529 medication records were analyzed. Overall, it was found that the formulary information in the EHR was accurate $93.1 \%$ of the time $(n=1,423 / 1,529)$, and the formulary information in Epocrates was accurate $89.4 \%$ of the time $(n=1,367 / 1,529$; Figure 1). The majority of medication records $(\mathrm{n}=1,227,80.2 \%)$ analyzed were for Medicaid patients. It was found that the formulary information in the EHR was more accurate (96.3\%) than Epocrates (88.2\%) for these patients. However, Epocrates was more accurate for patients with BCBS insurance (94.4\%) than the EHR (80.1\%).

\section{Pediatric Clinic}

A total of 936 medication records from the pediatric clinic were included in the analysis (Table 1). The majority of these patients had Medicaid insurance (88.4\%). For these patients, the EHR formulary information was more accurate than the formulary information found in Epocrates. There were a very small number of patients with BCBS insurance at the pediatric clinic (11.6\%). For these patients, the information in Epocrates was more accurate than the EHR. Overall, the most common medications prescribed were albuterol inhaler (ProAir HFA, $n=84)$, topical triamcinolone $(n=42)$, amoxicillin $(n=38)$, fluticasone inhalation $(n=35)$, albuterol nebulization $(n=32)$, and fluticasone nasal spray $(\mathrm{n}=31)$. The most common OTC medications prescribed were cetirizine $(n=54)$, polyethylene glycol $(n=31)$, topical hydrocortisone $(n=26)$, and clotrimazole $(n=17)$. Inaccuracies were more frequent with OTC products, and the most common incorrect formulary information was for cetirizine 5 milligram per milliliter $(\mathrm{mg} / \mathrm{mL})$ syrup $(\mathrm{n}=37)$, topical hydrocortisone $1 \%$ cream $(n=22)$, clotrimazole $1 \%$ cream $(n=17)$, and loratadine $(n=7$ [10 mg tablet, $n=5])$. The most common inaccurate prescription medications were epinephrine (Epi-Pen) injection ( $\mathrm{n}=5)$, aluminum chloride hexahydrate (Drysol) topical solution $(n=3)$, and various brandname prescriptions for methylphenidate ( $\mathrm{n}=2$ for Concerta 18 mg tablet and Daytrana $15 \mathrm{mg}$ transdermal patch).

\section{Family Medicine Clinic}

Of the 1,529 medication records, 593 (38.8\%) were for patients at the family medicine clinic. Most of the patients (67.5\%) had Medicaid insurance (Table 2). Again, it was found that for patients with Medicaid, the formulary information in the EHR was more accurate than that found in Epocrates, but for patients with BCBS, Epocrates was the most accurate. The most common medications prescribed were omeprazole $(n=20)$, albuterol inhaler (ProAir HFA, $n=18$ ), lisinopril $(n=18)$, metformin $(n=17)$, hydrochlorothiazide $(n=15)$, and pravastatin $(n=15)$. The most common OTC medications prescribed were cetirizine $(n=10)$, loratadine $(n=9)$, aspirin $(n=8)$, polyethylene glycol $(n=7)$, and vitamin D $(n=6)$. Similar to the pediatric clinic, the most frequent inaccuracies occurred with OTC products. The most common OTC products with incorrect formulary information were cetirizine $(\mathrm{n}=10)$ with the $10 \mathrm{mg}$ tablet being the most frequently prescribed $(n=6)$, loratadine $(\mathrm{n}=9$ [10 $\mathrm{mg}$ tablet, $\mathrm{n}=8]$ ), and aspirin $(\mathrm{n}=7$ [81 mg tablet, $n=3]$ ). There was not a consistent pattern for inaccuracies in prescription products, since such a wide variety are prescribed in family medicine. The most common inaccurate prescription 


\section{TABLE 1 Pediatric Clinic}

\begin{tabular}{|c|c|c|c|c|}
\hline \multirow{2}{*}{$\begin{array}{l}\text { Accurate } \\
\text { EHR, n (\%) }\end{array}$} & \multicolumn{2}{|c|}{ Medicaid $(n=827)$} & \multicolumn{2}{|c|}{ BCBS $(n=109)$} \\
\hline & 801 & $(96.9)$ & 91 & $(83.5)$ \\
\hline Epocrates, n (\%) & 716 & $(86.6)$ & 101 & $(92.7)$ \\
\hline
\end{tabular}

\section{TABLE 2 Family Medicine Clinic}

\begin{tabular}{|c|c|c|c|c|}
\hline Accurate & Medica & $(\mathrm{n}=400)$ & BCB & $1=193)$ \\
\hline EHR, n (\%) & 381 & (95.3) & 151 & (78.2) \\
\hline Epocrates, n (\%) & 366 & (91.5) & 184 & (95.3) \\
\hline
\end{tabular}

medications were brand-name esomeprazole (Nexium) $20 \mathrm{mg}$ capsules and $40 \mathrm{mg}$ capsules ( $\mathrm{n}=2$ for each) and generic azelastine 137 microgram/spray nasal solution $(n=3)$.

\section{Over-the-Counter Versus Prescription Medications}

Approximately 15\% of the medication records analyzed were for medications that are available OTC $(n=232)$. When these medication records were analyzed separately from prescriptiononly medications, it was found that overall the EHR was 90.5\% accurate, and Epocrates was $41.4 \%$ accurate. For Medicaid patients, the EHR was $93.6 \%$ accurate, and Epocrates was $38.1 \%$ accurate for OTC medications. For patients with BCBS, the EHR was $70 \%$ accurate, and Epocrates was $63.3 \%$ accurate for these patients. When OTC medications were removed from the analysis, the accuracy of Epocrates was much higher, ranging from 97.1 to $99.0 \%$. See Table 3 and Table 4 for clinicspecific information.

\section{Discussion}

The formulary information in the EHR system at our institution and in Epocrates was relatively reliable for our patient population. Overall, it was found that the EHR had the most accurate information for patients with Medicaid insurance, while Epocrates was more accurate for patients with BCBS. This difference was consistent across both clinics included in the study. The most discrepancies were found surrounding the coverage of OTC medications. For example, at the pediatric clinic, many patients were prescribed cetirizine oral syrup. Alabama Medicaid covers this medication for pediatric patients in spite of it being available OTC. However, Epocrates listed it as OTC and nonformulary. We hypothesized that this differentiation was the driving reason for the differences between the accuracy of each source for the 2 insurance providers. Medicaid covers more OTC medications (especially for pediatric patients), so Epocrates was incorrect more often for these patients than for those with BCBS. There were also a greater number of OTC medications prescribed for Medicaid patients
TABLE 3 Over-the-Counter Medication Coverage

\begin{tabular}{l|c|c|c|c}
\hline \multirow{2}{*}{} & \multicolumn{2}{|c|}{ Family Medicine Clinic } & \multicolumn{2}{c}{ Pediatric Clinic } \\
\cline { 2 - 5 } & Medicaid & BCBS & Medicaid & BCBS \\
\hline Total OTC medications, $\mathrm{n}$ & 52 & 21 & 150 & 9 \\
\hline EHR accurate, $\mathrm{n}(\%)$ & $47(90.4)$ & $13(61.9)$ & $142(94.7)$ & $8(88.9)$ \\
\hline Epocrates accurate, n (\%) & $25(48.1)$ & $17(81.0)$ & $52(34.7)$ & $2(22.2)$ \\
\hline $\begin{array}{l}\text { BCBS = Blue Cross Blue Shield; EHR=electronic health record; OTC = over the } \\
\text { counter. }\end{array}$
\end{tabular}

TABLE 4 Prescription Medication Coverage

\begin{tabular}{l|c|c|c|c}
\hline \multirow{2}{*}{} & \multicolumn{2}{|c|}{ Family Medicine Clinic } & \multicolumn{2}{c}{ Pediatric Clinic } \\
\cline { 2 - 5 } & Medicaid & BCBS & Medicaid & BCBS \\
\hline $\begin{array}{l}\text { Total prescription } \\
\text { medications, } \mathrm{n}\end{array}$ & 348 & 172 & 677 & 100 \\
\hline EHR accurate, $\mathrm{n}(\%)$ & $334(96.0)$ & $138(80.2)$ & $659(97.3)$ & $83(83.0)$ \\
\hline Epocrates accurate, $\mathrm{n}(\%)$ & $341(98.0)$ & $167(97.1)$ & $664(98.1)$ & $99(99.0)$ \\
\hline BCBS = Blue Cross Blue Shield; EHR=electronic health record.
\end{tabular}

(17\% of total medications prescribed for Medicaid patients vs. $9.9 \%$ for BCBS patients). The most common medications prescribed in both clinics matched the most common disease states treated in the patient populations. The OTC allergy medications were the most common OTC products prescribed in both clinics, likely due to the peak of fall seasonal allergies during our study period. Also, the most commonly prescribed OTC products were the most common products with formulary inaccuracies. There were no consistent patterns for inaccuracies in prescription products, which corresponds to our findings that formulary information for prescription medications is generally much more accurate than that of OTC products in our EHR and in Epocrates.

Our study was the first study analyzing the accuracy of these 2 sources of formulary information in this patient population. A strength of this study is the large number of medication records analyzed. We also analyzed patient medication records at 2 separate clinics, increasing the diversity of patients and types of medications prescribed. Data were collected over several months and included 2 Medicaid formulary updates.

\section{Limitations}

There were several potential limitations in our study. There was a small number of patients with BCBS insurance compared with Medicaid due to the demographics of the patients seen in the 2 clinics. Also, there are many different insurance plans available through BCBS. The insurance information in the EHR did not specify each patient's specific plan. A general BCBS plan was used to determine the formulary status of each medication. Each specific plan follows the same tiered system but may have a few unique exclusions or limitations. Epocrates also did not distinguish between different BCBS plans. Another 
limitation is that Epocrates does not distinguish between different strengths of some medications that are available both OTC and prescription-only. For example, topical hydrocortisone is listed as OTC, although some strengths are only available by prescription. Also, physicians were not surveyed to assess which resources were used for formulary information, and prescribing practices based on the outcomes of this study were not measured.

\section{Conclusions}

We found that formulary information generated by the EHR system at our institution and the Epocrates application can be useful tools for prescribers. Insurance coverage and patient cost are important aspects to consider when prescribing medications, since high medication cost has been shown to lead to decreased patient adherence. Epocrates and our EHR allow physicians to access formulary information quickly and use it as part of the decision-making process when choosing a medication for a patient. However, it is important to remember that neither source is completely accurate. In our patient population, the EHR was more reliable for patients with Medicaid insurance, while Epocrates was more accurate for patients with BCBS. For medications that are available OTC, prescribers should be very cautious, and if insurance coverage is a concern, online posted formularies should be consulted.

\section{Authors}

MIRANDA R. ANDRUS, PharmD, BCPS, FCCP, is Associate Clinical Professor of Pharmacy Practice, and LEA S. EILAND, PharmD, BCPS, FASHP, is Clinical Professor of Pharmacy Practice, Auburn University Harrison School of Pharmacy, Huntsville, Alabama. JEANNE B. FORRESTER, PharmD, is PGY1 Pharmacy Resident, The Medical University of South Carolina, Charleston, and KENDA E. GERMAIN, PharmD, is PGY1 Pharmacy Resident, Mission Hospital, Asheville, North Carolina.

AUTHOR CORRESPONDENCE: Miranda R. Andrus, PharmD, BCPS, FCCP, Associate Clinical Professor of Pharmacy Practice, Auburn University Harrison School of Pharmacy, 301 Governors Dr., Huntsville, AL 35803. Tel.: 256.551.4458;

E-mail: andrumr@auburn.edu.

\section{DISCLOSURES}

No author has any financial interest or affiliations with the medications, databases, or electronic health record discussed in this article. The research for this study was not funded. All authors substantially contributed to the research project and manuscript development. All authors approved the final version of the manuscript submitted.

Study concept and design were contributed by Eiland and Andrus. Forrester and Germain collected the data, which were interpreted by all authors equally. The manuscript was written and revised by Andrus, with the help of the rest of the authors.

\section{REFERENCES}

1. Shrank W, Hoang T, Ettner S, et al. The implications of choice: prescribing generic or preferred pharmaceuticals improves medication adherence for chronic conditions. Arch Intern Med. 2006;166(3):332-37. Available at: http://archinte.jamanetwork.com/article.aspx?articleid=409766. Accessed December 18, 2014.

2. McMullin ST, Lonergan TP, Rynearson CS. Twelve-month drug cost savings related to use of an electronic prescribing with integrated decision support in primary care. J Manag Care Spec Pharm. 2005;11(4):322-32. Available at: http://www.amcp.org/WorkArea/DownloadAsset.aspx?id=7157.

3. Lapane K, Rosen R, Dubé C. Perceptions of e-prescribing efficiencies and inefficiencies in ambulatory care. Int J Med Inform. 2011;80(1):39-46.

4. Fischer M, Vogeli C, Stedman M, Ferris T, Brookhart A, Weissman JS. Effect of electronic prescribing with formulary decision support on medication use and cost. Arch Intern Med. 2008;168(22):2433-39. Available at: http://archinte.jamanetwork.com/article. aspx?articleid=773454. Accessed December 18, 2014.

5. Ross SM, Papshev D, Murphy EL, Sternberg DJ, Taylor J, Barg R. Effects of electronic prescribing on formulary compliance and generic drug utilization in the ambulatory care setting: a retrospective analysis of administrative claims data. J Manag Care Spec Pharm. 2005;11(5):410-15. Available at: http:// www.amcp.org/data/jmcp/contemporary_410-415.pdf.

6. Crosson J, Schueth A, Isaacson N, Bell DS. Early adopters of electronic prescribing struggle to make meaningful use of formulary checks and medication history documentation. J Am Board Fam Med. 2012;25(1):24-32. Available at: http://www.jabfm.org/content/25/1/24.full.pdf+html. Accessed December 18, 2014

7. Wang CJ, Patel MH, Schueth AJ, et al. Perceptions of standards-based electronic prescribing systems as implemented in outpatient primary care: a physician survey. J Am Med Inform Assoc. 2009;16(4):493-502. Available at: http://www.ncbi.nlm.nih.gov/pmc/articles/PMC2705252/. Accessed December 18, 2014

8. Medicare Program; Standards for e-prescribing under Medicare Part D and identification of backward compatible version of adopted standard for e-prescribing and the Medicare prescription drug program (Version 8.1). 42 CFR Part 423 (2008). Available at: http://www.gpo.gov/fdsys/pkg/FR-200804-07/pdf/08-1094.pdf. Accessed December 18, 2014.

9. Conrad Joshua L (Director of Patient Safety and Clinical Utility, Epocrates). E-mail to Jeanne Forrester (Auburn University, Huntsville, AL) February 25, 2014.

10. Alabama Medicaid Agency. Medicaid primer. February 2012. Available at: http://www.medicaid.alabama.gov/documents/2.0_Newsroom/2.1_ About_Medicaid/2.1_Medicaid_Primer_10-12-12.pdf. Accessed December 18,2014

11. Alabama Medicaid Agency. Report of the Alabama Medicaid Pharmacy Study Commission. December 2013. Available at: http://www.medicaid. alabama.gov/documents/2.0_Newsroom/2.2_Boards_Committees/2.2.2_ Pharmacy_Study_Commission/2.2.2_AL_Medicaid_Pharmacy\%20 Commission_Report_12-30-13.pdf. Accessed December 18, 2014.

12. Alabama Medicaid Agency. Co-payments for Medicaid services. June 2013. Available at: http://216.226.177.83/documents/4.0_Programs/4.2_ Covered_Services/4.2_Co_Payments_Handout_6-28-13.pdf. Accessed December 18, 2014.

13. Blue Cross Blue Shield of Alabama. Company overview. Available at: https://www.bcbsal.org/employers/about/overview.cfm. Accessed December 18,2014

14. Blue Cross Blue Shield of Alabama. Blue Cross and Blue Shield of Alabama tiered generic prescription drug guide. October 2014. Available at: https://www.myprime.com/content/dam/prime/memberportal/forms/2014/ FullyQualified/Other/ALL/BCBSAL/COMMERCIAL/ALSEIBCOMM/AL_ Tiered_Prescription_Drug_Guide.pdf. Accessed December 18, 2014. 